\title{
Reverse chromosome painting: a method for the rapid analysis of aberrant chromosomes in clinical cytogenetics
}

\author{
N P Carter, M A Ferguson-Smith, $M$ T Perryman, H Telenius, A H Pelmear, \\ M A Leversha, M T Glancy, S L Wood, K Cook, H M Dyson, \\ M E Ferguson-Smith, L R Willatt
}

\begin{abstract}
We describe a method, termed reverse chromosome painting, which allows the rapid analysis of the content and breakpoints of aberrant chromosomes. The method involves the sorting of small numbers of the aberrant chromosome from short term blood culture preparations or cell lines by using bivariate flow karyotype analysis. The sorted chromosomes are amplified and biotin labelled enzymatically using a degenerate oligonucleotide-primed polymerase chain reaction (DOP-PCR), the product annealed to metaphase spreads from normal subjects, and hybridisation detected using fluorescence in situ hybridisation (FISH). We show the usefulness of this method for routine clinical cytogenetics by the analysis of cases involving an insertion, $a$ deletion, a translocation, and two cases of a chromosome with additional material of unknown origin. The method has particular application for the rapid resolution of the origin of de novo unbalanced chromosome duplications.
\end{abstract}

Department of Pathology, University of Cambridge, Tennis Court Road, Cambridge CB2 1QP. N P Carter

M A Ferguson-Smith

M T Perryman

A $\mathrm{H}$ Pelmear

M A Leversha

East Anglian Regional Genetics Service,

Addenbrooke's

Hospital, Hills Road,

Cambridge.

M A Ferguson-Smith

M T Glancy

$S$ L Wood

K Cook

H M Dyson

$M$ E Ferguson-Smith

L R Willatt

\section{CRC Human Cancer}

Genetics Research

Group, Department of

Pathology, University

of Cambridge, and

Department of

Clinical Genetics,

Karolinska Hospital,

Stockholm, Sweden.

$\mathrm{H}$ Telenius

Correspondence to

Dr Carter.

Received 9 December 1991

Accepted 6 January 1992. unknown origin, the only approach is each library in turn until hybridisation of the abnormal chromosome is observed. ${ }^{6}$ An additional limitation of the traditional chromosome painting approach in such cases is that sub- chromosomal region information about the source of the additional material is not obtained. Furthermore, deletions cannot be visualised by hybridisation of chromosome libraries onto the abnormal chromosome.

In this paper we describe a reverse chromosome painting technique in which the probe is generated from the aberrant chromosome itself and applied to metaphase spreads of normal subjects using fluorescence in situ hybridisation (FISH), so highlighting the constituents of the aberrant chromosome directly on normal chromosomes. Briefly, small numbers of the aberrant chromosome are flow sorted from a routine peripheral blood culture and amplified in a general way using a degenerate oligonucleotide primed polymerase chain reaction (DOP-PCR) protocol. ${ }^{7}$ This complex probe is biotinylated during secondary DOP-PCR cycles and then hybridised onto normal metaphase spreads using CISS. Detection by avidin-fluorescein isothiocyanate conjugates enables chromosome regions present in the aberrant chromosome to be visualisd on the normal metaphase chromosomes by using fluorescence microscopy. Recently we have shown that this approach can be used for the analysis of the breakpoints of translocated chromosomes sorted from cell lines. ${ }^{7}$ In this paper, we show that the reverse chromosome painting approach is readily applicable to clinical cytogenetic practice, without the need for establishment of cell lines, by reporting the analysis of five diagnostic cases involving an insertion, a deletion, a translocation, and two cases with additional chromosomal material of unknown origin.

\section{Methods}

\section{CELL PREPARATIONS}

Phytohaemagglutinin stimulated peripheral blood cultures $(0.5 \mathrm{ml}$ of blood in $8 \mathrm{ml}$ medium) were established and metaphase spreads were prepared using standard techniques with overnight synchronisation using methotrexate and release with bromodeoxyuridine. ${ }^{8}$ Routine cytogenetic analysis was performed on GTL banded preparations. Slides prepared for in situ hybridisation were allowed to air dry and then fixed further in methanol: acetic acid (3:1) for one hour. These slides were then dehydrated through a $70 \%, 90 \%$, and $100 \%$ ethanol series, fixed in acetone for 10 minutes, and allowed to air dry. Slides were

.


stored desiccated at room temperature for between seven and 14 days before use.

For flow analysis, one or two blood cultures as above were blocked after 72 or 96 hours with $0 \cdot 1 \mu \mathrm{g} / \mathrm{ml}$ colcemid (BRL) overnight. Leucocytes were separated by centrifugation $(800 \mathrm{~g}$ for 20 minutes) over a density step gradient of Lymphopaque 1119 (Sigma). Cells at the density interface were removed, pooled if necessary, and washed twice in $20 \mathrm{ml}$ of medium (RPMI 1640, 16\% fetal calf serum, $2 \mathrm{mmol} / \mathrm{l} \mathrm{L}$-glutamine, penicillin $100 \mathrm{units} / \mathrm{ml}$, streptomycin $100 \mathrm{mg} / \mathrm{ml}$, colcemid $0.1 \mu \mathrm{g} / \mathrm{ml}$ ) by centrifugation ( $200 \mathrm{~g}$ for eight minutes), resuspended in $2 \mathrm{ml}$ of hypotonic buffer $(75 \mathrm{mmol} / 1 \quad \mathrm{KCl}, \quad 0.2 \mathrm{mmol} / 1$ spermine, $0.5 \mathrm{mmol} / 1$ spermidine), and allowed to swel for 15 minutes at room temperature. After centrifugation for five minutes at $400 \mathrm{~g}$, the cell pellet was resuspended in $380 \mu$ l of polyamine buffer ( $15 \mathrm{mmol} / 1$ tris(hydroxymethyl)methylamine, $0.2 \mathrm{mmol} / 1 \mathrm{spermine}, 0.5 \mathrm{mmol} / 1 \mathrm{sper}$ midine, $2 \mathrm{mmol} / 1 \mathrm{EDTA}, 0.5 \mathrm{mmol} / 1 \mathrm{EGTA}$, $80 \mathrm{mmol} / 1 \mathrm{KCl}, 20 \mathrm{mmol} / 1 \mathrm{NaCl}, 14 \mathrm{mmol} / \mathrm{l}$ $\beta$-mercaptoethanol, $0 \cdot 25 \%$ Triton-X 100, $\mathrm{pH}$ $7 \cdot 2$ ), and incubated on ice for 10 minutes. The suspension was then vortexed for 10 to 15 seconds to release the chromosomes into suspension. The chromosomes were stained by the addition of $40 \mu \mathrm{g} / \mathrm{ml}$ of Chromomycin A3, $2 \mathrm{mmol} / 1 \mathrm{magnesium}$ sulphate, and $2 \mu \mathrm{g} / \mathrm{ml}$ of Hoechst 33258 and incubated for at least two hours. Ten minutes before flow analysis, sodium citrate and sodium sulphite were added to a final concentration of $10 \mathrm{mmol} / \mathrm{l}$ and $25 \mathrm{mmol} / 1$ respectively to give a final suspension volume of $500 \mu \mathrm{l}$.

\section{FLOW CYTOMETRY}

Chromosome preparations were analysed on a FACStar Plus flow sorter (Becton Dickinson) equipped with two $5 \mathrm{~W}$ argon ion lasers as described previously. ${ }^{9}$ Between 300 and 400 specific chromosomes were sorted directly into $500 \mu \mathrm{l}$ PCR tubes containing $33 \mu \mathrm{l}$ of pure water and then stored at $4^{\circ} \mathrm{C}$.

\section{DOP-PCR AMPLIFICATION}

DOP-PCR amplification from the sorted chromosomes was performed as described previously. ${ }^{7}$ Briefly, reaction buffer $(25 \mathrm{mmol} / \mathrm{l}$ $\mathrm{N}$-tris (hydroxymethyl) methyl-3-amino-proanesulfonic acid, $50 \mathrm{mmol} / 1 \mathrm{KCl}, 2 \mathrm{mmol} / 1$ $\mathrm{MgCl}_{2}, 1 \mathrm{mmol} / \mathrm{l}$ dithiothreitol, $\mathrm{pH} \mathrm{9.0),} \mathrm{de-}$ tergent $(0.05 \%$ polyoxyethylene ether $\mathrm{W}-1)$, dinucleotide triphosphates $(200 \mu \mathrm{mol} / 1$ of each triphosphate), primer 6-MW ( $5^{\prime}$ CCG ACT CGA GNN NNN NAT GTG G 3' where $\mathrm{N}=$ any base, $2 \mu \mathrm{mol} / \mathrm{l}$ ) and 2.5 units of Taq polymerase (NBS Biologicals) were added from concentrated stocks to give a final reaction volume of $50 \mu \mathrm{l}$, which was overlayered with $30 \mu \mathrm{l}$ of mineral oil. After an initial denaturation for nine minutes at $94^{\circ} \mathrm{C}$, five cycles of $94^{\circ} \mathrm{C}$ for one minute, $30^{\circ} \mathrm{C}$ for 1.5 minutes, transition at $0.23^{\circ} \mathrm{C} / \mathrm{sec}$ to $72^{\circ} \mathrm{C}$ held for three minutes were followed immediately by 35 cycles of $94^{\circ} \mathrm{C}$ for one minute, $62^{\circ} \mathrm{C}$ for one minute, and $72^{\circ} \mathrm{C}$ for three minutes. The final extension at $72^{\circ} \mathrm{C}$ was increased to 10 minutes. Biotinylation was achieved by subjecting $300 \mathrm{ng}$ of primary PCR product in reaction buffer as above but supplemented with dUTP11 -biotin $(0.28 \mathrm{mmol} / 1$ final concentration,
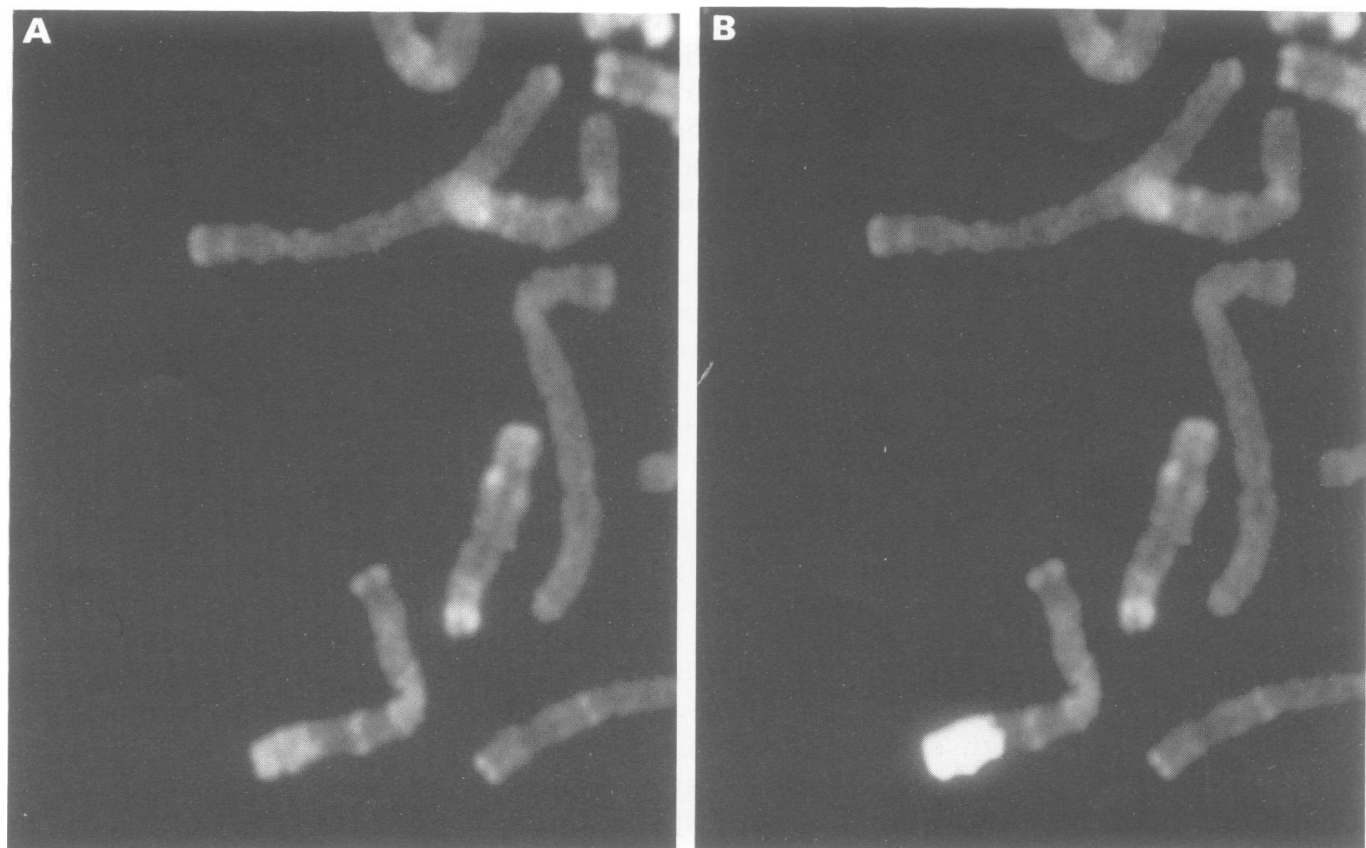

Figure 1 Localisation of hybridisation signal on fluorescent reverse banded chromosomes (normal chromosomes, paint from case 3). (A) Image from detector 1 (propidium iodide fluorescence) of the confocal microscope showing reverse bands. (B) Image from detector 2 (fluorescein isothiocyanate fluorescence) merged with image from detector 1 showing localisation of hybridisation from $931.2 \rightarrow$ qter on chromosome 7 . 
Sigma) to further amplification (initial denaturation at $94^{\circ} \mathrm{C}$ for three minutes followed by 25 cycles of $94^{\circ} \mathrm{C}$ for one minute, $62^{\circ} \mathrm{C}$ for 2 minutes, and $72^{\circ} \mathrm{C}$ for three minutes, final extension at $72^{\circ} \mathrm{C}$ increased to 10 minutes). All PCR reactions were performed on a Trio thermal cycler (Biometra) and product concentration was determined using a TKO 100 DNA fluorometer (Hoefer).

CISS HYBRIDISATION AND PROBE DETECTION

Hybridisations and detection were carried out using a modification of the procedure described by Pinkel et al. ${ }^{4}$ Briefly, for each slide, $50 \mathrm{ng}$ of probe and $500 \mathrm{ng}$ Cot 1 DNA (BRL) were made up to $20 \mu \mathrm{l}$ with hybridisation buffer $^{10}(50 \% \mathrm{v} / \mathrm{v}$ deionised formamide, $10 \%$ w/v dextran sulphate, $2 \times$ SSC, $40 \mathrm{mmol} / 1$

\section{Case 1}

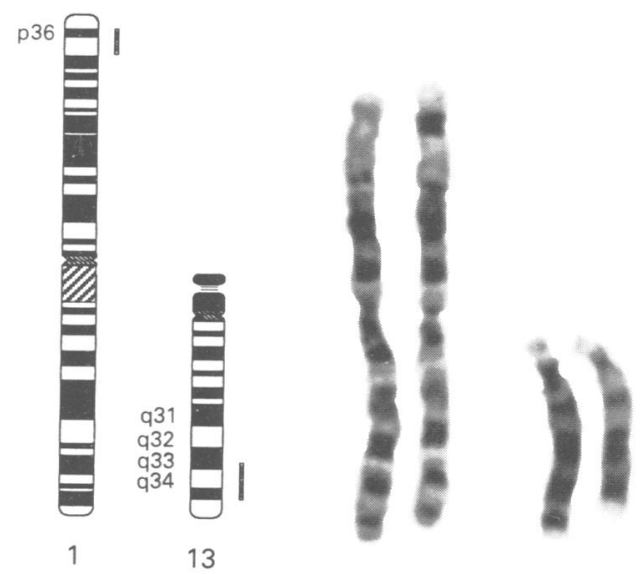

Case 2
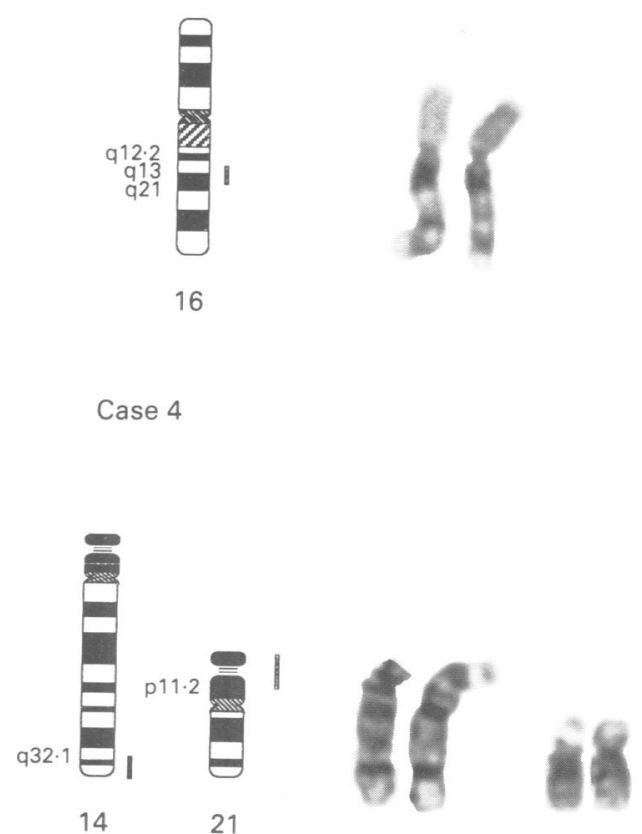

Figure 2 Partial karyotypes by GTL banding. Bars indicate approximat is on the right of each pair. For detailed explanation of each case refer to text. sodium phosphate, $0 \cdot 1 \%$ SDS, $1 \times$ Denhardt's solution, $\mathrm{pH} 7 \cdot 0.1 \times$ Denhardt's solution $=$ $0.02 \%$ polyvinylpyrrolidone, $0.02 \%$ Ficoll, $0.02 \%$ bovine serum albumin. $1 \times \mathrm{SSC}=$ $0.15 \mathrm{~mol} / 1 \mathrm{NaCl}, 15 \mathrm{mmol} / 1$ sodium citrate, $\mathrm{pH}$ 7.5), mixed well, denatured for 10 minutes at $65^{\circ} \mathrm{C}$, and preannealed for one hour at $37^{\circ} \mathrm{C}$. Slides were denatured in $70 \%$ formamide, $2 \times$ SSC for exactly two minutes at $65^{\circ} \mathrm{C}$, quenched in ice cold $70 \%$ ethanol, dehydrated through a $70 \%, 90 \%$, and $100 \%$ ethanol series, and air dried. The preannealed probe was applied to slides and allowed to hybridise overnight at $42^{\circ} \mathrm{C}$. After stringency washes in $50 \%$ formamide, $1 \times$ SSC, hybridisation was detected by incubation with avidin-FITC DCS (Vector Labs). The signal was usually amplified by further incubation with biotinylated anti-avidin (Vector Labs) followed 
by avidin-FITC. Chromosomes were counterstained and reverse banded by mounting the slides in Citifluor antifade AF1 (Citifluor Ltd) containing $2.5 \mu \mathrm{g} / \mathrm{ml}$ of DAPI and $0.5 \mu \mathrm{g} / \mathrm{ml}$ of propidium iodide. Hybridised slides were assessed using a Nikon Optiphot fluorescence microscope with a $100 \times, 1.4$ na objective and an I2 filter block. Images were recorded as grey levels using both detectors (detector 1 , 500 to $560 \mathrm{~nm}$; detector $2<600 \mathrm{~nm}$ ) of an MRC 600 confocal scanning head (Biorad) and displayed merged in pseudocolour (FITC fluorescence in green, PI fluorescence in red). The band location of FITC signal was determined by toggling the FITC signal on and off to allow the banding pattern beneath the signal to be displayed (fig 1).

\section{Results}

CASE 1

The patient was a 33 year old female, para $2+2$ (the two fetal losses were terminations of pregnancies with neural tube defects). The first live born was a normal male; however, the second was a male with unilateral coloboma of the iris and choroid, a possible midline brain defect identified on scan, and hypotonia.

Chromosome analysis of cultured lymphocytes from the second male child showed an abnormal karyotype, 46,XY,13q-. Maternal chromosomes showed an interstitial deletion/ insertion involving chromosomes 1 and 13, with a segment around band $\mathrm{q} 33$ on the long arm of chromosome 13 inserted into p36 on the short arm of chromosome 1 (fig 2). The karyotype was interpreted as $46, \mathrm{XX}$,ins $(1 ; 13)(\mathrm{p} 36 ; \mathrm{q} 32 \mathrm{q} 34)$. It was noted that band

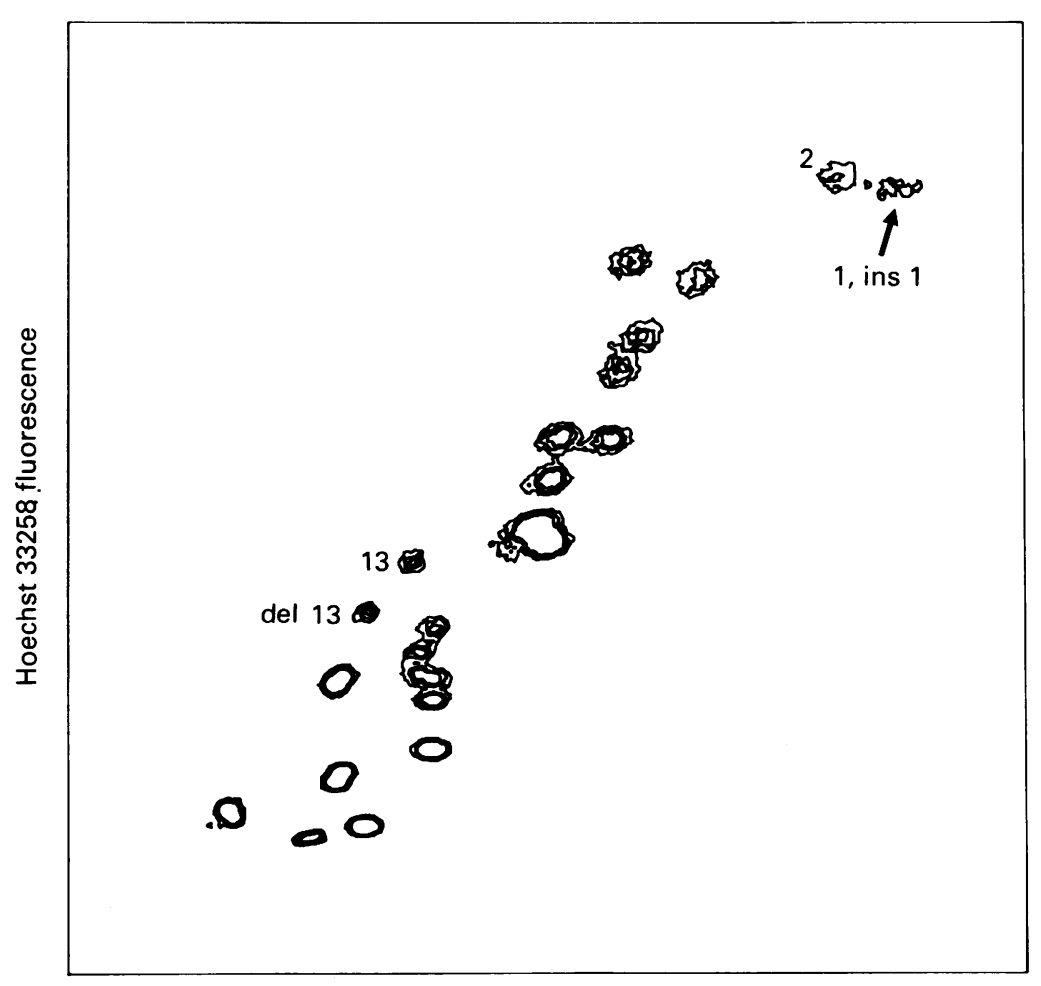

Chromomycin A3 fluorescence

Figure 3 Maternal flow karyotype from case 1 (see text for details). The peak containing the normal and derivative chromosomes 1 is indicated. 13q33 appeared to resolve into two bands when inserted into chromosome 1 and various interpretations were considered.

From the maternal flow karyotype (fig 3), it can be seen that the chromosome 13 with the deletion is readily resolved but that the chromosome 1 with the insertion could not be separated from the normal chromosome 1. Therefore, it was necessary to sort both of these chromosomes 1 to produce the paint involving the inserted chromosome. A typical normal male metaphase hybridised with this paint is shown in fig 4 . In addition to the two normal chromosomes 1 , signal was detected on the graphics display from distal 13q31, through 13q32, and involving a large part of 13q33. These breakpoints were confirmed by analysis of the paint derived from the chromosome with the deletion (data not shown). The origin and position of the insertion was confirmed when a normal chromosome 13 paint was hybridised to the patient's chromosomes (data not shown).

CASE 2

The proband, a 1 year old girl, was referred for routine cytogenetic investigation from the local Child Development Centre. She had small hands, unusual facies, and general developmental delay. Chromosome analysis of cultured lymphocytes showed a female karyotype with an interstitial deletion in the long arm of one chromosome 16 involving q13 to q21 (karyotype 46,XX, $\operatorname{del}(16)$ (pter $\rightarrow$ q13::q21 $\rightarrow$ qter)). The small $G$ dark band present between $\mathrm{q} 12$ and $\mathrm{q} 21$ on the derivative chromosomes (fig 2) can only be explained by breakpoints distal in $16 \mathrm{q} 13$ and distal in 16q21. Parental karyotypes were apparently normal.

Despite a poor yield of cells and a low mitotic index resulting in a flow karyotype of reduced resolution (fig 5), the deleted chromosome 16 could be distinguished easily and was sorted for PCR amplification. A typical normal metaphase hybridised with the derivative paint is shown in fig $4 \mathrm{~B}$. While most of the length of the chromosomes 16 is painted, signal is missing from the centromere and the region involving distal 16q13 and all of 16q21.

CASE 3

A 33 year old expectant mother (para $0+1$ ) was referred for prenatal diagnosis by chorionic villus sampling (CVS) at 11 weeks' gestation. Her partner's karyotype showed an apparently balanced reciprocal translocation, $46, X Y, t(7 ; 21)(q 31 ; q 22)$, identified through an extensive family study of recurrent miscarriages. The CVS analysis showed the fetus to be female with the apparently balanced reciprocal translocation, $46, \mathrm{XX}, \mathrm{t}(7 ; 21)(\mathrm{q} 31 ; \mathrm{q} 22)$. At term a phenotypically normal female baby was born and the karyotype was confirmed by chromosome analysis from a cord blood sample taken at delivery (fig 2).

The flow karyotype of the child is shown in fig 6 . The $t(7 ; 21)$ is confirmed by half the 

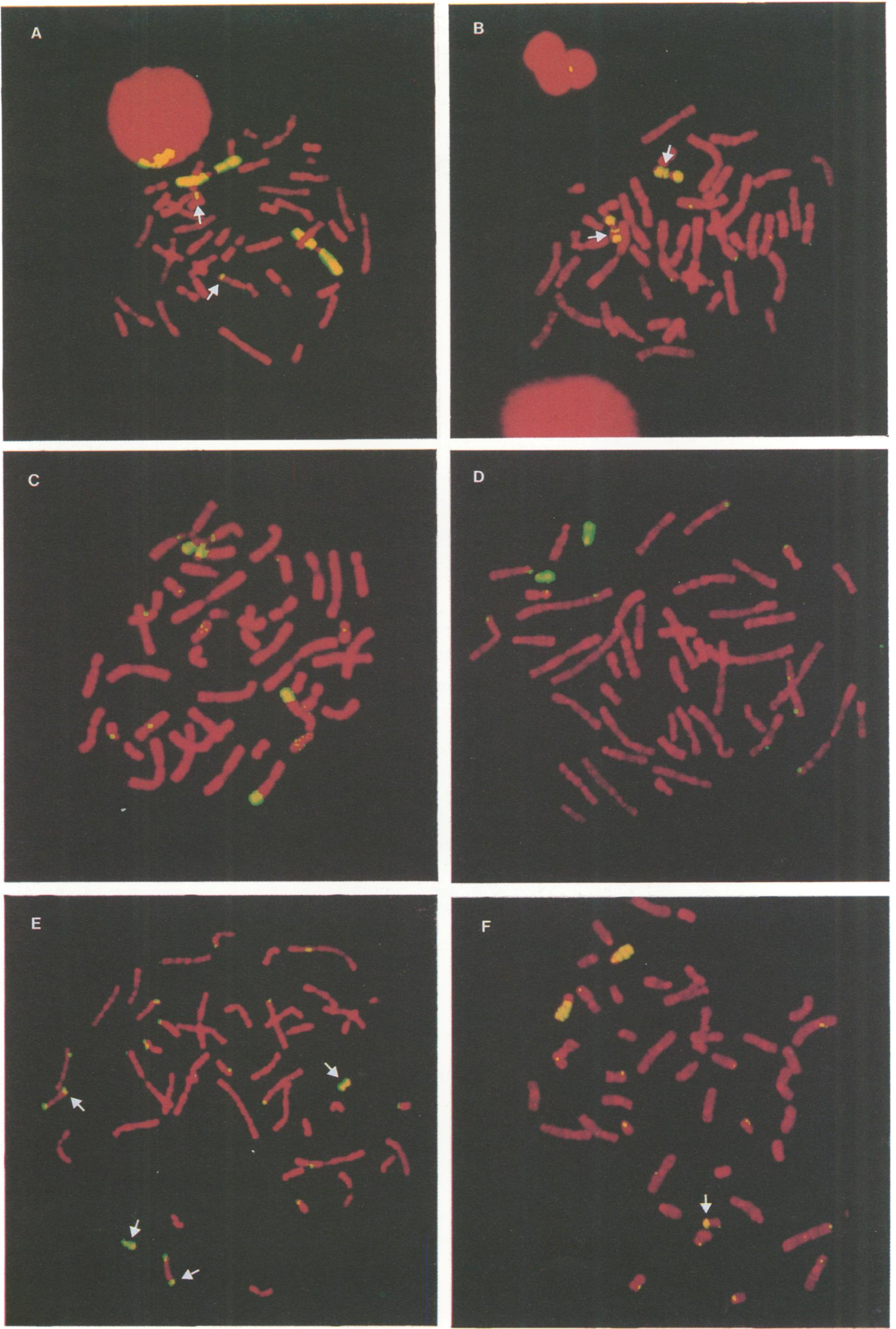

Figure 4 Reverse and conventional chromosome painting analyses of cases 1 to 4 (see text for details). (A) Chromosome paint derived from the normal and derivative chromosomes 1 in case 1 hybridised onto a normal metaphase spread. The chromosome 13 material present in the derivative chromosome 1 is visualised on the two normal chromosomes 13 (arrowed). (B) Chromosome paint derived from the chromosome 16 with the deletion in case 2 hybridised onto a normal metaphase spread. The region of chromosome 16 deleted in the patient is represented by the region on the long arms of the normal chromosomes 16 which are not painted (arrowed). (C) Chromosome paint derived from the derivative chromosome 21 in case 3 hybridised onto a normal metaphase spread. The regions of chromosomes 7 and 21 involved in the translocation are visualised directly. (D) Chromosome paint from the normal chromosome 21 in case 4 hybridised onto a normal metaphase spread. Only the two normal chromosomes 21 are painted. (E) Chromosome paint from the derivative chromosome 21 in case 4 hybridised onto a normal metaphase spread. As well as signals on the two normal chromosomes 21 (arrowed) and acrocentric short arms, region

932.1 $\rightarrow$ qter of the normal chromosomes 14 are painted. (F) Chromosome paint from sorted normal chromosome 14 hybridised onto a metaphase spread of the patient in case 4. As well as the two chromosomes 14, the distal part of the short arm of the derivative chromosome 21 is painted. 


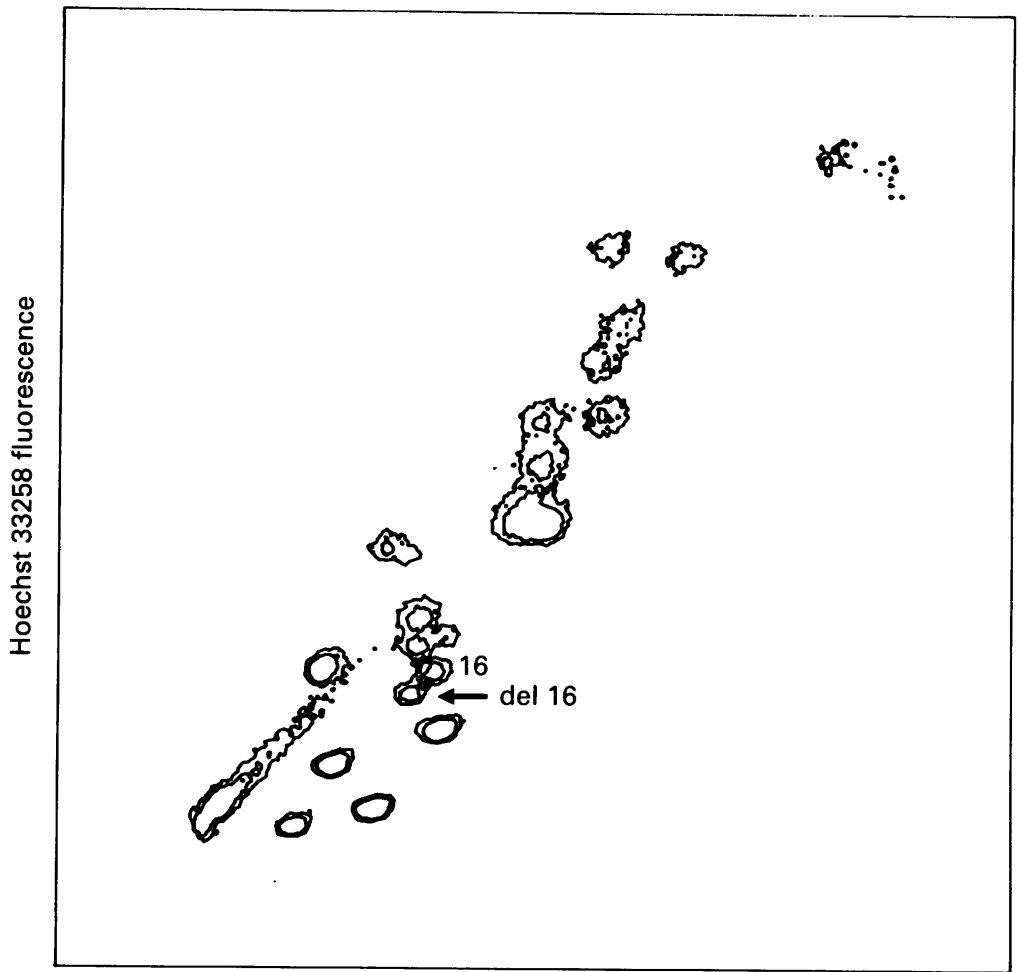

Chromomycin A3 fluorescence

Figure 5 Flow karyotype from case 2. The peaks representing the normal and derivative chromosomes 16 are indicated.

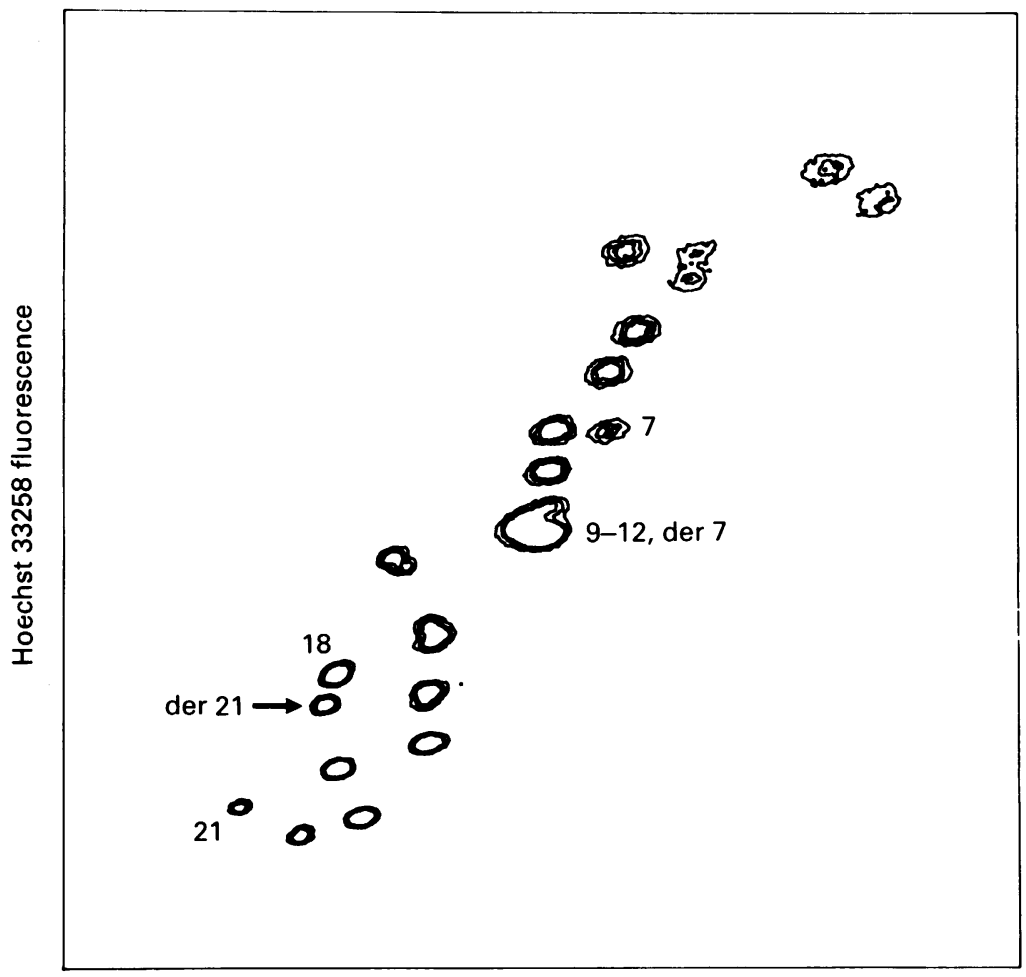

Chromomycin A3 fluorescence

Figure 6 Flow karyotype from case 3. The peaks representing the normal and derivative chromosomes 7 and 21 are indicated.

normal number of events in the chromosome 7 and chromosome 21 peaks. The derivative 21 is resolved clearly just below chromosome 18 while the derivative 7 is contained within the chromosome 9 to 12 cluster. Therefore only the derivative 21 could be sorted for PCR amplification. A typical normal male meta- phase hybridised with the derivative 21 paint is shown in fig 4C. The two chromosomes 7 show signal from q31.2 $\rightarrow$ qter, while the two chromosomes 21 show signal from $\mathrm{q} 11.2 \rightarrow \mathrm{q} 22.2$.

CASE 4

The proband, a 1 year old boy, was referred for routine cytogenetic investigation from the local outpatient department. He had hypotonia and delayed motor development. Chromosome analysis of cultured lymphoctyes showed a male karyotype, with additional material on the short arm of one chromosome 21 (karyotype $46, X Y, 21 p+$, fig 2 ). As the short arm of the aberrant chromosome 21 was AgNOR and $C$ band negative, we were unable to determine the origin of the additional euchromatic material using the conventional cytogenetic approach. Parental karyotypes were normal, confirming that this unbalanced chromosome duplication had occurred de novo.

The flow karyotype of this boy is shown in fig 7. As well as heteromorphisms of chromosomes 13,20 , and 22 , the normal chromosome 21 and the derivative chromosome 21 are resolved clearly. Both the normal chromosome 21 and derivative chromosome 21 were sorted separately for amplification by PCR. The paint generated from the normal chromosome 21 showed hybridisation only to chromosomes 21 on a normal male metaphase spread (fig 4D). However, the derivative chromosome 21 paint showed hybridisation on normal metaphase spreads to chromosomes 21 , acrocentric short arms, and to the region $\mathrm{q} 32.1 \rightarrow \mathrm{qter}$ of chromosomes 14 (fig $4 \mathrm{E}$ ). From this analysis the child has a duplication of chromosome 14 involving region $\mathrm{q} 32.1 \rightarrow \mathrm{qter}$. In this case the diagnosis of chromosome 14 as the source of the additional material in the derivative chromosome 21 was confirmed by hybridising metaphases from the child with a DOP-PCR paint generated from normal chromosomes 14 sorted from a lymphoblastoid cell line. Fig $4 \mathrm{~F}$ shows an example of this conventional painting analysis where both chromosomes 14 and the distal part of the short arm of one chromosome 21 display fluorescent signal.

CASE 5

The patient, a 6 year old girl tall for her age, was referred for investigation because of developmental delay and mild dysmorphic features. Chromosome analysis of cultured lymphocytes (fig 2) showed a female karyotype with additional material on the short arm of chromosome 8 distal to $8 \mathrm{p} 22$ (karyotype $46, \mathrm{XX}, 8 \mathrm{p}+$ ). As a result of the addition, the region $8 \mathrm{p} 22 \rightarrow$ pter was estimated to be approximately twice the normal size. However, the chromosomal origin of the extra material could not be determined by GTL banding. Both parents have apparently normal karyotypes.

The flow karyotype of the patient is shown in fig 8. The derivative chromosome 8 was found to lie between chromosomes $\mathrm{X}$ and 7 on the flow karyotype. A small sorting gate was 


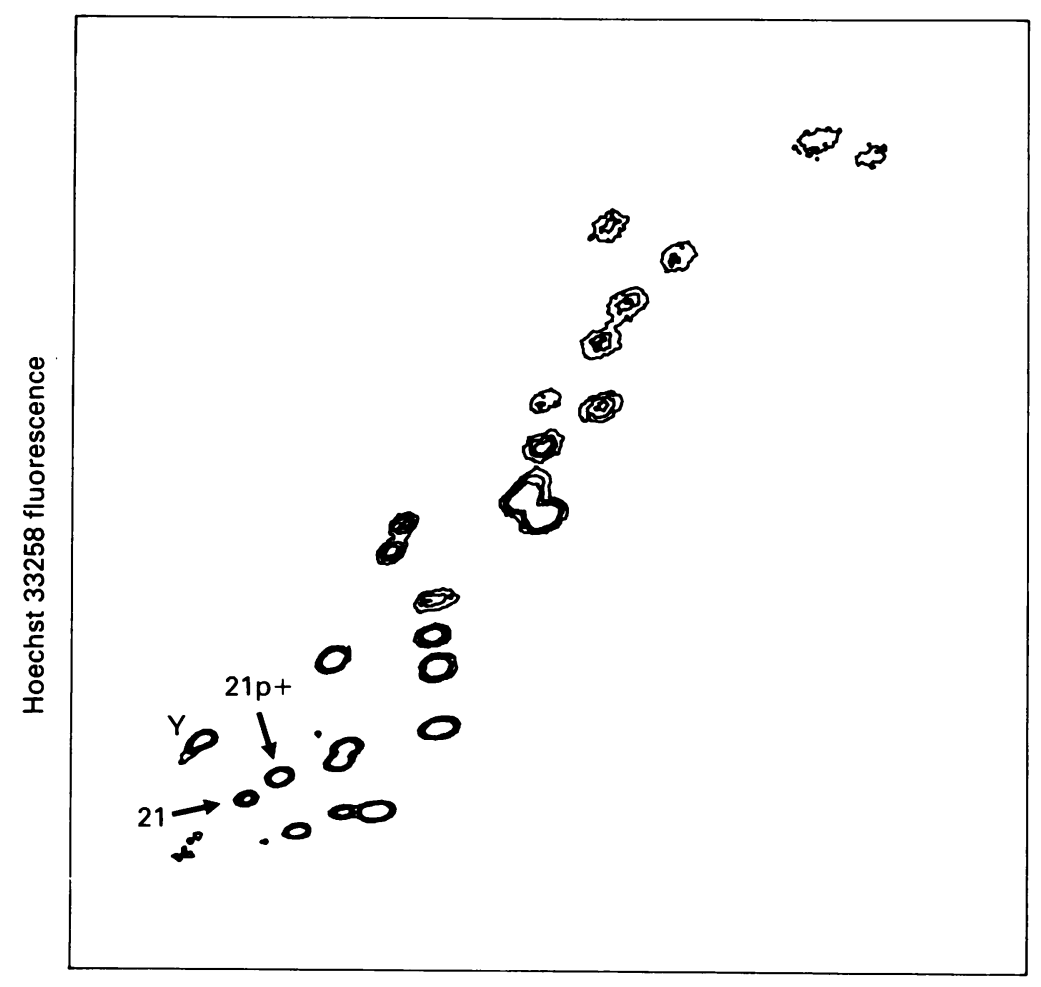

Chromomycin A3 fluorescence

Figure 7 Flow karyotype from case 4. The peaks representing the normal and derivative chromosomes 21 are indicated.

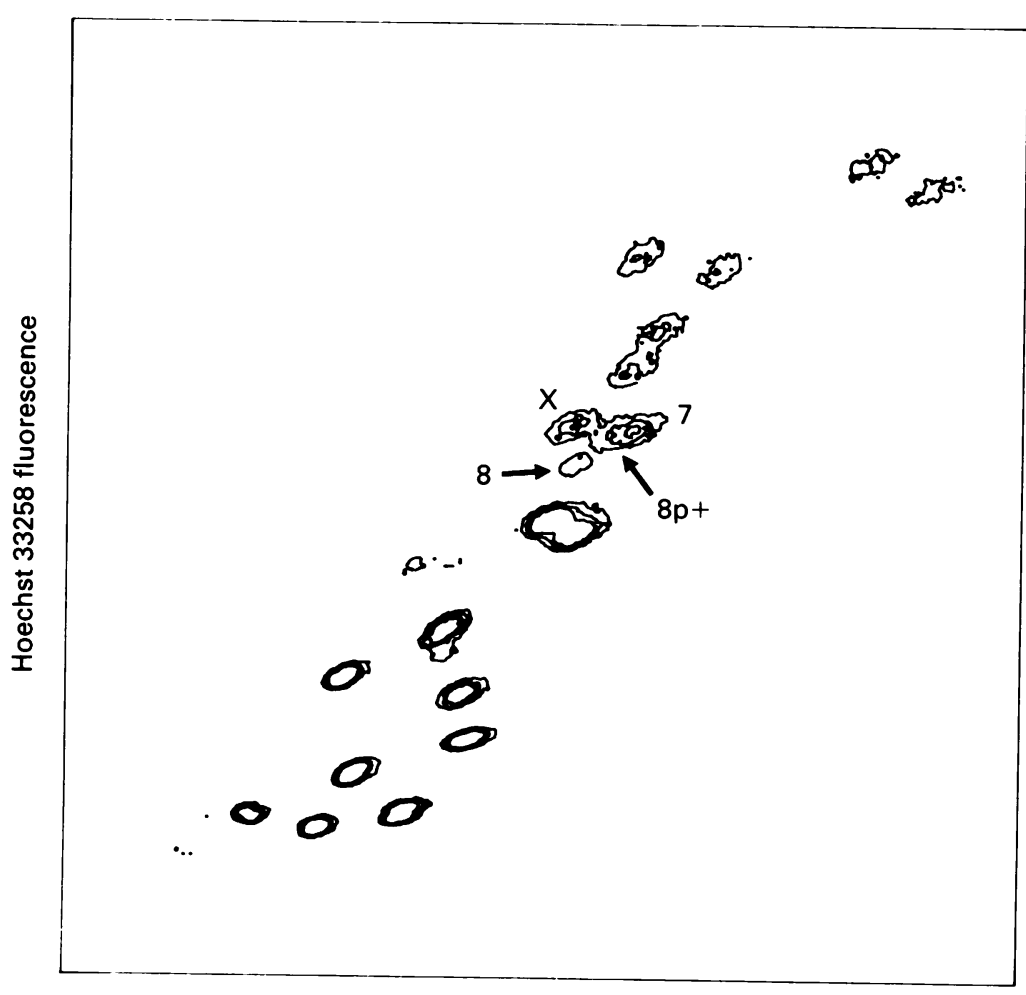

Chromomycin A3 fluorescence

Figure 8 Flow karyotype from case 5. The peaks representing the normal chromosomes $X, 8$, and 7 , and the derivative chromosome 8 are indicated.

set around this region in an attempt to include as little of chromosomes $\mathrm{X}$ and 7 as possible but contamination with these chromosomes was inevitable. The paint generated from this sort shows strong hybridisation to the chromosomes 8 on metaphase spreads of a normal male (fig 9A) with weaker hybridisation to chromosomes $\mathrm{X}$ and 7 . In particular, there was no evidence of incomplete painting of the normal chromosomes 8 as might be expected if the abnormal chromosome 8 from the patient was the result of a reciprocal translocation. As hybridisation to chromosomes $\mathrm{X}$ and 7 was expected from the impure nature of the flow sorting and as no signals other than those on chromosomes 8 were detected, we concluded that the derivative chromosome 8 was the result of a duplication of chromosome 8 . This conclusion was tested by hybridising the patient's metaphases with a normal chromosome 8 paint. It can be seen from fig 9B that the entire length of both the normal and the aberrant chromosomes 8 are painted, so confirming the intrachromosomal origin of the aditional chromosomal material.

\section{Discussion}

In this study we have shown that reverse chromosome painting, where the paint is generated rapidly from small numbers of flow sorted aberrant chromosomes and applied to normal metaphase spreads, is a useful technique for the analysis of abnormal karyotypes. Reverse chromosome painting is clearly the method of choice for determining the origin of de novo unbalanced chromosome duplications (for example cases 4 and 5) which cannot be resolved by conventional banding techniques. It is encouraging to note that in all but one case, the specific band assignments found by cytogenetic analysis and the reverse painting technique were consistent.

In case 1, cytogenetic analysis indicated an insertion of $13 \mathrm{q} 33$ into $1 \mathrm{p} 36$ although the precise location of the insertion into 1 p 36 could not be determined by conventional banding. The reverse painting showed the involvement of a region including part of distal $13 q 31$, all of 13q32, and most of 13q33. Allowing for the modification of $G$ band staining at the site of translocation breakpoints, the insertion of this region above $1 \mathrm{p} 35$ is consistent with the banding pattern of the derivative chromosomes in that an enlarged $G$ dark band at 1 p35 (1p35 plus 13q31) would be produced with an additional $G$ dark band derived from the distal part of $13 \mathrm{q} 33$ present below $1 \mathrm{p} 36.1$ (fig 2). Thus, the consensus karyotype for this subject becomes $46, \mathrm{XX}$,dir ins $(1 ; 13)(1 \mathrm{pter} \rightarrow$ $1 \mathrm{p} 36.1:: 13 \mathrm{q} 33 \rightarrow 13 \mathrm{q} 31:: 1 \mathrm{p} 36.1 \rightarrow$ qter; $13 \mathrm{pter} \rightarrow$ $13 q 31:: 13 q 33 \rightarrow 13 q$ ter)

In case 3, cytogenetic analysis of the translocation showed breakpoints at bands $7 q 31$ and $21 \mathrm{q} 22$, but more detailed analysis was not possible as we were unable to determine, even at high banding resolution, whether $21 \mathrm{q} 22.2$ was translocated. However, it was clear from the reverse painting that the breakpoints were $7 \mathrm{q} 31.2$ and distal $21 \mathrm{q} 22.2$.

Analysis of case 4 showed immediately that the additional chromosomal material on $21 \mathrm{p}$ was derived from the region $\mathrm{q} 32.1 \rightarrow \mathrm{qter}$ of chromosome 14 . This result shows the power of the reverse chromosome painting technique 

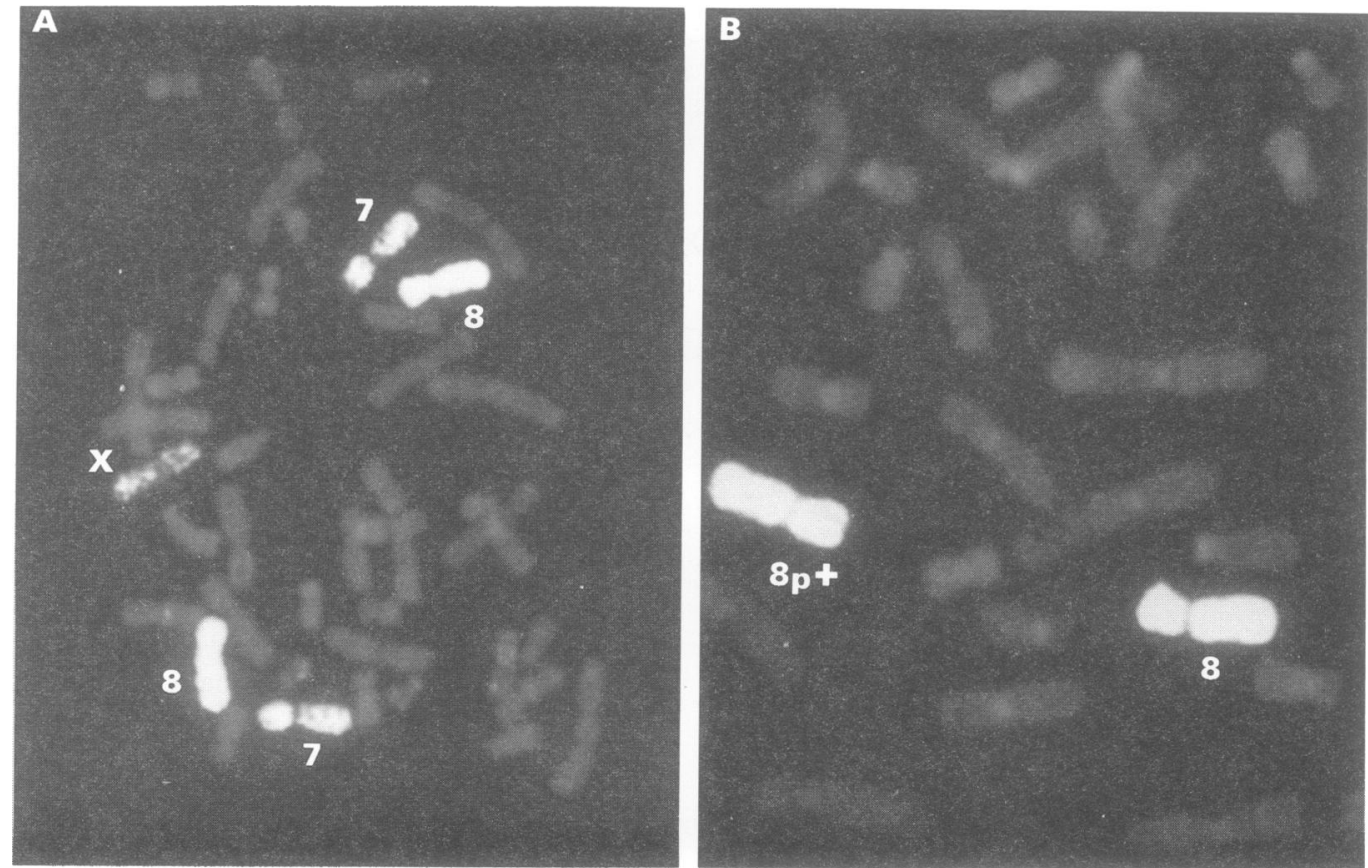

Figure 9 Chromosome painting analysis of case 5. (A) Chromosome paint derived from the flow sorting region between chromosomes $X$ and 7 (to include the derivative chromosome 8 ) in case 5 hybridised onto a normal metaphase spread. Strong signals can be seen on the normal chromosomes 8 with weaker signals on chromosomes $X$ and 7. (B) Chromosome paint from sorted normal chromosome 8 hybridised onto a metaphase spread of the patient in case 5. The entire length of the derivative chromosome 8 is painted confirming the chromosome 8 origin of the duplication.

for cases of this type as both the chromosomal origin and subchromosomal localisation of the de novo duplication is visualised directly. Conventional hybridisation onto the patient's chromosomes with a normal chromosome 14 paint (as in fig 4F) indicated that this additional material was indeed derived from chromosome 14, but cannot provide information as to which region of chromosome 14 was involved. In hindsight, it can be seen in fig 2 that the small $\mathrm{G}$ dark band in the short arm of the derivative chromosome 21 (which did not silver stain) is consistent with the presence of band $14 \mathrm{q} 32.2$.

The diagnosis of an intrachromosomal duplication was made by reverse painting in case 5 where, once again, the source of the additional material arising de novo on $8 p$ was not evident on conventional banding. In this case, while the chromosome 8 origin of the duplication is clear, it is not possible to determine the regional localisation of the duplication by reverse painting. Regional chromosome paints made from sorting appropriate translocation derivatives should help to resolve this difficulty. Once the duplication is localised, chromosome 8 specific probes derived from the region could be used to define its extent more accurately. Nevertheless, this case shows the value of the method for the initial detection of intrachromosomal aberrations.

Only in case 2 was there slight disagreement in breakpoint assignment between the two analysis techniques. The GTL banding pattern on the long arm of the derivative chromosome shown in fig 2 can only be explained by the loss of distal $16 \mathrm{q} 13$ and most but not all of $16 q 21$. While reverse painting showed that the proximal end of the deletion occurred distal in
$16 \mathrm{q} 13$ in agreement with banding analysis, all of band 16q21 appeared to be lost. One explanation for this discrepancy could be the difference between band positions of GTL banded and RBF banded preparations. Alternatively, although the visualisation of breakpoints using the reverse banding technique appears to be remarkably specific even on relatively short chromosomes, the fluorescence complex itself lies above the chromosome and some positional error is likely to occur. The amount of complex formed, which is dependent on probe and detection reagent concentrations, will determine the degree of spreading of the signal around the hybridisation site. Another important factor is the setting of the gain and threshold of the detection hardware, as it is possible by using inappropriate settings for fluorescence signal to spread out from the specific hybridisation site. These factors, while controlled in this study, can all lead to a slight overestimation of the length of a painted section of a chromosome and thus may provide inaccurate breakpoint analysis unless this point is appreciated.

Some other limitations to the usefulness of the reverse chromosome painting technique should be mentioned. The DOP-PCR amplification, although producing relatively even signal on the euchromatin of chromosomes, often fails to paint highly repetitive sequences in acrocentric short arms, at the centromere, and in some heterochromatic regions. This can be seen in fig 4 where centromeres and in some cases acrocentric short arms are not painted. This effect is either because of the failure of these sequences to amplify (the DOP-PCR amplification is specific for the most $3^{\prime}$ six base sequence) (Telenius $e t a l$, in preparation) or, 
more likely, because of complete suppression of the signal by the Cot 1 DNA. However, whether these repetitive regions hybridise or not is unpredictable and varies from chromosome amplification to chromosome amplification. Thus, in case 3, the paint produced from the amplified derivative chromosome 21 (which contains $21 \mathrm{p}$ ) did not hybridise to $21 \mathrm{p}$ on the normal chromosomes. However, in case 4 , sequences from $21 \mathrm{p}$ were amplified from the $21 p+$ chromosome so that the short arms of the normal chromosomes 21 were painted. A further complication in this latter case is that acrocentric short arms share the same repetitive sequences so that the generated paint will hybridise not only to $21 \mathrm{p}$ but also to the other acrocentric short arms. This is particularly noticeable in fig $4 \mathrm{D}$. This complication in the variable amplification of such repetitive chromosome regions must be taken into account in the interpretation of reverse painting analyses.

Another consideration is the accuracy and purity of the initial chromosome sorting from which the paint is generated. When the derivative chromosome is resolved on the flow karyotype away from the other normal chromosomes, a paint specific for the aberrant chromosome can be produced and a straightforward analysis performed. However, in some cases (for example cases 1 and 5), the derivative chromosomes will overlie others or be so close as to preclude pure sorting. However, in this event, it is still possible to sort the region containing the aberrant chromosome and generate a complex paint of both the aberrant and co-sorting normal chromosomes. Most often, the co-sorting normal chromosome will not be involved in the derivation of the aberrant chromosome and a reverse painting analysis will still be possible (for example, cases 1 and 5), but care in the interpretation of such cases must be exercised. Another limitation is found in the analysis of insertions. Reverse chromosome painting can readily identify the chromosome and region of the material involved in the insertion, but cannot identify where in the derivative chromosome the insertion has taken place. Here conventional painting, using a flow sorted library or DOP-PCR product specific for the donor chromosome type, can be used to identify on the inserted chromosome itself the location of the insertion.

It should be mentioned that, while the reverse painting technique is readily applied to long term cultures (and we have had particular success with immortalised lymphoblastoid cell lines), our aim here is to show the application of the method to routine short term cultures from peripheral blood samples. Although fluorescence activated chromosome sorting may not be readily available to all chromosome diagnostic laboratories, a cost effective chromosome sorting service could be established by a small number of supraregional laboratories. Only a comparatively small proportion of abnormal cases encountered by a diagnostic laboratory would be appropriate for reverse painting.

As shown here, the technique is most useful for determining the nature of de novo unbalanced chromosome duplications and for determining the extent of deletions. Our experience with small marker chromosomes is limited at present to two cases with mosaicism for a tiny marker chromosome less than one third the size of chromosome 21 . In both cases, the extra chromosome did not produce a distinctive peak on the flow karyotype made from peripheral blood cultures and so could not be sorted. We do not envisage such problems with larger marker chromosomes as these have been resolved previously using lymphoblastoid cell lines. ${ }^{11}$ In fact, reverse painting may well be the method of choice in such cases.

The reverse painting technique shows promise in the interpretation of high resolution banding analysis where a particular pattern may be explained by several different combinations of breakpoints ${ }^{12}$ (for example, case 1). Altogether, the combined techniques of high resolution banding, reverse painting, and conventional painting provide a powerful resource for diagnostic cytogenetics in the future.

This work was carried out with support from the Medical Research Council. We are grateful to $G$ van den Engh for the program ANALIST which was used to produce the flow karyotype contour plots.

1 Cremer T, Lichter P, Borden J, et al. Detection of chromosome aberrations in metaphase and interphase tumour cells by in-situ hybridization using chromosome-specific library probes. Hum Genet 1988;80:235-46.

2 Lichter P, Cremer T, Borden J, et al. Delineation of individual human chromosomes in metaphase and interphase cells by in-situ suppression hybridization using recombinant DNA libraries. Hum Genet 1988;80:224-34.

3 Lichter P, Cremer T, Chang Tang CJ, et al. Rapid detection of chromosome 21 aberrations by in-situ hybridization. Proc Natl Acad Sci USA 1988;85:9664-8.

4 Pinkel D, Landegent J, Collins C, et al. Fluorescence in situ hybridization with human chromosome-specific libraries: detection of trisomy 21 and translocations of chromosome 4. Proc Natl Acad Sci USA 1988;85:9138-42.

5 Jauch A, Oaumer C, Lichter P, et al. Chromosomal in-situ suppresson hybridization of human gonosomes and autosomes and its use in clinical cytogenetics. Hum Genet 1990;85:145-50.

6 Hulten MA, Gould CP, Goldman ASH, et al. Chromosome in situ suppression hybridisation in clinical cytogenetics. f Med Genet 1991;28:577-82.

7 Telenius H, Pelmear AH, Tunnacliffe A, et al. Cytogenetic analysis by chromosome painting using DOP-PCR amplified flow-sorted chromosomes. Genes, Chromosomes and Cancer 1992;4:257-63.

8 Rooney DE, Czepulkowski BH. Human cytogenetics: a practical approach. Oxford: IRL Press, 1986.

9 Carter NP, Ferguson-Smith ME, Affara NA, et al. Study of $\mathrm{X}$ chromosome abnormality in XX males using bivariate flow karyotype analysis and flow sorted dot blots. Cyto-

metry 1990;11:202-7.
10 Viegas-Pequignot E, Dutrillaux B, Magdelenat $\mathrm{H}$, et al. Mapping of single-copy DNA sequences on human chromosomes by in situ hybridisation with biotinylated probes: enhancement of detection sensitivity by intensified-fluorescence digital-imaging microscopy. Proc Nat Acad Sci USA 1989;86:582-6.

11 Waters JJ, Ferguson-Smith ME, Carter NP, et al. Prenatal diagnosis of a double bisatellited marker with an unusual copy number ratio. Prenat Diagn 1990;10:677-81.

12 Savage JRK. Assignment of aberration breakpoints in banded chromosomes. Nature 1977;270:513-4. 